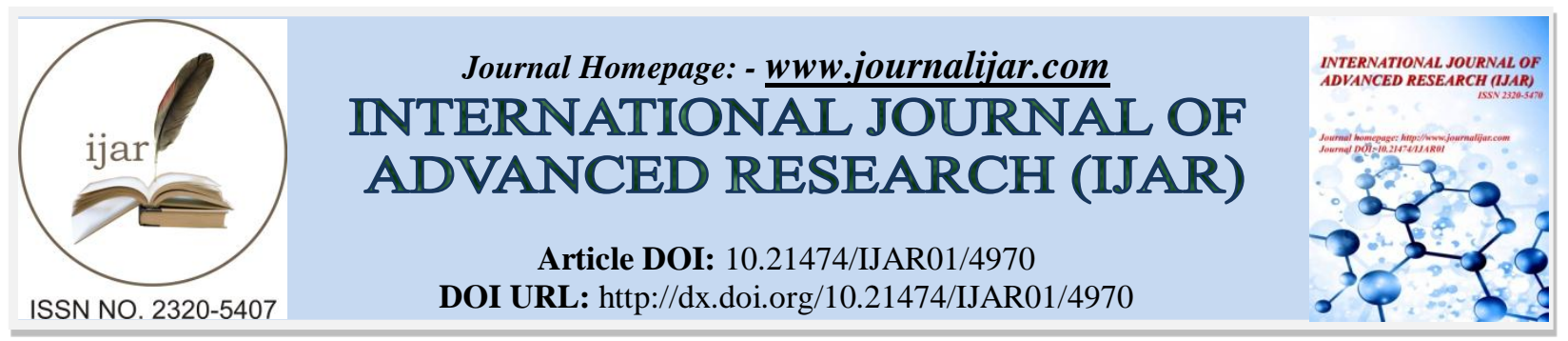

RESEARCH ARTICLE

\title{
SOME SEMI-REGULAR WEAKLY CONTINUOUS FUNCTIONS IN TOPOLOGICAL SPACES.
}

*R. S. Wali, Basayya B. Mathad ${ }^{1}$ and Nirani Laxmi'

1. Department of Mathematics, Bhandari \& Rathi College, Guledagudd-587 203, Karnataka, India.

2. Department of Mathematics, Rani Channamma University, Belagavi-591 156, Karnataka, India.

\section{Manuscript Info}

Manuscript History

Received: 26 May 2017

Final Accepted: 28 June 2017

Published: July 2017

Key words:-

srw-continuous, srw-irresolute, perfectly srw-continuous, and strongly srw-

continuous functions.

\section{Abstract}

The authors introduced semi-regular weakly closed sets and semiregular weakly open sets in topological spaces and established their relationships with some generalized sets in topological spaces. The purpose of this paper is to introduce semi-regular weakly continuous (briefly srw-continuous), semi-regular weakly irresolute (briefly srwirresolute), perfectly semi-regular weakly continuous (briefly perfectly srw-continuous) and strongly semi-regular weakly continuous (briefly strongly srw-continuous) functions by using srw-closed and srw-open sets properties and also we discuss their basic properties.

Copy Right, IJAR, 2017,. All rights reserved.

\section{Introduction:-}

In 1963 N. Levine [7] introduced semi-continuous functions using semi-open sets. Balachandran et. al. [2] introduced the concepts of generalized continuous functions and gc-irresolute functions on topological spaces. Recently R.S. Wali et. al. [11] introduced $\alpha$ rw-continuous continuous functions and also several authors I. Arockiarani [1], R. Devi et. al. [4], J. Dontchev and Maki [5], A. S. Mashhour et. al. [8] and N. Palaniappan and K. C. Rao [9] worked in the field of general topology and have shown interest in studying the concept of generalizations of continuous functions.

In this paper, we introduce new class of continuous functions called srw-continuous, srw-irresolute, perfectly srwcontinuous and strongly srw-continuous functions in topological spaces and also we discuss their properties and characteristics.

\section{Preliminaries:-}

Throughout this paper $(\mathrm{X}, \tau),(\mathrm{Y}, \sigma)$ and $(\mathrm{Z}, \gamma)$ or simply $\mathrm{X}, \mathrm{Y}$ and $\mathrm{Z}$ will always denote topological spaces on which no separation axioms are assumed unless explicitly stated. int(A), cl(A), srw-cl(A), and srw-int(A) denote the interior of A, closure of A, srw-closure of A and srw-interior of A respectively. X\A denotes the complement of A in $\mathrm{X}$. We recall the following definitions and results.

Definition2.1 A subset A of a topological space $X$ is called

i) Regular open [11], if $\mathrm{A}=\operatorname{int}(\mathrm{cl}(\mathrm{A}))$ and regular closed if $\operatorname{cl}(\operatorname{int}(\mathrm{A}))=\mathrm{A}$.

ii) Pre-open [11], if $\mathrm{A} \subseteq \operatorname{int}(\operatorname{cl}(\mathrm{A}))$ and pre-closed if $\operatorname{cl}(\operatorname{int}(\mathrm{A})) \subseteq \mathrm{A}$.

iii) Semi open [11], if $\mathrm{A} \subseteq \operatorname{cl}(\operatorname{int}(\mathrm{A}))$ and semi-closed if $\operatorname{int}(\mathrm{cl}(\mathrm{A}) \subseteq \mathrm{A}$.

iv) $\alpha$-open [11], if $\subseteq \subseteq \operatorname{int}(\operatorname{cl}(\operatorname{int}(\mathrm{A})))$ and $\alpha$-closed if $\operatorname{cl}(\operatorname{int}(\operatorname{cl}(\mathrm{A})) \subseteq \mathrm{A}$.

v) Semi pre open [11], if $\mathrm{A} \subseteq \operatorname{cl}(\operatorname{int}(\operatorname{cl}(\mathrm{A})))$ and semi pre closed if $\operatorname{int}(\operatorname{cl}(\operatorname{int}(\mathrm{A}))) \subseteq \mathrm{A}$. 
vi) $\pi$-open [11], if $\mathrm{A}$ is a finite union of regular open sets.

The semi-pre-closure (resp. semi-closure, resp. pre-closure, resp. $\alpha$-closure) of a subset $\mathrm{A}$ of $\mathrm{X}$ is the intersection of all semi-pre-closed (resp. semi-closed, resp. pre-closed, resp. $\alpha$-closed) sets containing A and is denoted by spcl(A) (resp. $\operatorname{scl}(\mathrm{A}), \operatorname{resp} . p c l(\mathrm{~A})$, resp. $\alpha \mathrm{cl}(\mathrm{A})$ ).

Definition 2.2 A subset A of a topological space $\mathrm{X}$ is called

i) Generalized closed (briefly g-closed) [11], if cl(A) $\subseteq \mathrm{U}$ wheneverA $\subseteq \mathrm{U}$ and $\mathrm{U}$ is open in $\mathrm{X}$.

ii) Generalized semi-closed (briefly gs-closed) [11], if $\operatorname{scl}(\mathrm{A}) \subseteq \mathrm{U}$ whenever $\mathrm{A} \subseteq \mathrm{U}$ and $\mathrm{U}$ is open in $\mathrm{X}$.

iii) Generalized $\alpha$-closed (briefly g $\alpha$-closed) [11], if $\alpha \operatorname{cl}(\mathrm{A}) \subseteq \mathrm{U}$ whenever $\mathrm{A} \subseteq \mathrm{U}$ and $\mathrm{U}$ is $\alpha$-open in $\mathrm{X}$.

iv) $\alpha$-generalized closed (briefly $\alpha$ g-closed) [11], if $\alpha \mathrm{cl}(\mathrm{A}) \subseteq \mathrm{U}$ whenever $\mathrm{A} \subseteq \mathrm{U}$ and $\mathrm{U}$ is open in $\mathrm{X}$.

v) Generalized semi pre-closed (briefly gsp-closed) [11], if $\operatorname{spcl}(\mathrm{A}) \subseteq \mathrm{U}$ whenever $\mathrm{A} \subseteq \mathrm{U}$ and $\mathrm{U}$ is open in $\mathrm{X}$.

vi) Regular generalized closed (briefly rg-closed) [11], if $\operatorname{cl}(\mathrm{A}) \subseteq \mathrm{U}$ whenever $\mathrm{A} \subseteq \mathrm{U}$ and $\mathrm{U}$ is regular open in $\mathrm{X}$.

vii) Weakly closed (briefly w-closed) [11], if cl(A) $\subseteq \mathrm{U}$ whenever $\mathrm{A} \subseteq \mathrm{U}$ and $\mathrm{U}$ is semi-open in $\mathrm{X}$.

viii) Regular weakly closed (briefly rw-closed) [11], if cl(A) $\subseteq \mathrm{U}$ whenever $\mathrm{A} \subseteq \mathrm{U}$ and $\mathrm{U}$ is regular semi-open in $\mathrm{X}$. ix) $\alpha$-regular weakly closed (briefly $\alpha$ rw-closed) [12], if $\alpha \mathrm{cl}(\mathrm{A}) \subseteq \mathrm{U}$ whenever $\mathrm{A} \subseteq \mathrm{U}$ and $\mathrm{U}$ is rw-open set in X. The complements of above all closed sets are their respective open sets in the same topological space X.

Definition 2.3 A subset A of a space $X$ is said to be semi regular weakly closed (briefly srw-closed) set [10], if $\operatorname{scl}(\mathrm{A}) \subseteq \mathrm{U}$ whenever $\mathrm{A} \subseteq \mathrm{U}$ and $\mathrm{U}$ is rw-open set in $\mathrm{X}$.

Definition 2.4 A subset A of $X$ is called semi regular weakly open (briefly srw-open) set [11], if $X \backslash A$ is srw-closed set in X.

Definition 2.5 For a subset $A$ of a space $X, \operatorname{srw}-\operatorname{cl}(A)=\cap\{F: A \subseteq F$ and $F$ is srw - closed set in $X\}$ is called srw-closure of $\mathrm{A}[11]$.

Definition 2.5 A function $f:(X, \tau) \rightarrow(Y, \sigma)$ is called

i) Completely continuous [3], if $f^{-1}(\mathrm{~V})$ is regular closed set of $(\mathrm{X}, \tau)$ for every closed set $\mathrm{V}$ of $(\mathrm{Y}, \sigma)$.

ii) g-continuous [3], if $f^{-1}(V)$ is g-closed set of $(X, \tau)$ for every closed set $V$ of $(Y, \sigma)$.

iii) semi-continuous [3], if $\mathrm{f}^{-1}(\mathrm{~V})$ is semi-closed set of $(\mathrm{X}, \tau)$ for every closed set $\mathrm{V}$ of $(\mathrm{Y}, \sigma)$.

iv) $\alpha$-continuous [3], if $\mathrm{f}^{-1}(\mathrm{~V})$ is $\alpha$-closed set of $(\mathrm{X}, \tau)$ for every closed set $\mathrm{V}$ of $(\mathrm{Y}, \sigma)$.

v) $\alpha$ g-continuous [3], if $f^{-1}(V)$ is $\alpha g$-closed set of $(X, \tau)$ for every closed set $V$ of $(Y, \sigma)$.

vi) gs-continuous [3], if $f^{-1}(V)$ is gs-closed set of $(X, \tau)$ for every closed set $V$ of $(Y, \sigma)$.

vii) gsp-continuous [3], if $f^{-1}(V)$ is gsp-closed set of $(X, \tau)$ for every closed set $V$ of $(Y, \sigma)$.

viii) $\alpha \mathrm{rw}$-continuous [12], if $\mathrm{f}^{-1}(\mathrm{~V})$ is $\alpha \mathrm{rw}$-closed set of $(\mathrm{X}, \tau)$ for every closed set $\mathrm{V}$ of $(\mathrm{Y}, \sigma)$.

ix) $w$-continuous [3], if $f^{-1}(V)$ is $w$-closed set of $(X, \tau)$ for every closed set $V$ of $(Y, \sigma)$.

Definition 2.6 A function $\mathrm{f}:(\mathrm{X}, \tau) \rightarrow(\mathrm{Y}, \sigma)$ is called

i) Irresolute [6], if $f^{-1}(V)$ is semi-open set of $(X, \tau)$ for every semi-open set $V$ of $(Y, \sigma)$.

ii) $w$-irresolute $[6]$, if $f^{-1}(V)$ is w-closed set of $(X, \tau)$ for every w-closed set $V$ of $(Y, \sigma)$.

iii) gc-irresolute [6], if $f^{-1}(V)$ is g-open set of $(X, \tau)$ for every g-open set $V$ of $(Y, \sigma)$.

iv) $\alpha$ rw-irresolute [12], if $f^{-1}(V)$ is $\alpha$ rw-closed set of $(X, \tau)$ for every $\alpha$ rw-closed set $V$ of $(Y, \sigma)$.

v) $\alpha$-irresolute [6], if $\mathrm{f}^{-1}(\mathrm{~V})$ is $\alpha$-open set of $(\mathrm{X}, \tau)$ for every $\alpha$-open set $\mathrm{V}$ of $(\mathrm{Y}, \sigma)$.

vi) gsp-irresolute [6], if $f^{-1}(V)$ is gsp-closed set of $(X, \tau)$ for every gsp-closed set $\mathrm{V}$ of $(\mathrm{Y}, \sigma)$.

vii) $g^{*}$-irresolute [6], if $f^{-1}(V)$ is $g^{*}$-open set of $(X, \tau)$ for every $g^{*}$-open set $V$ of $(Y, \sigma)$.

viii) rg-irresolute [6], if $\mathrm{f}^{-1}(\mathrm{~V})$ is rg-open set of $(\mathrm{X}, \tau)$ for every rg-open set $\mathrm{V}$ of $(\mathrm{Y}, \sigma)$.

ix) gpr-irresolute $[6]$, if $f^{-1}(V)$ is gpr-open set of $(X, \tau)$ for every gpr-open set $V$ of $(Y, \sigma)$.

$\mathrm{x})$ Perfectly-continuous [6], if $\mathrm{f}^{-1}(\mathrm{~V})$ is clopen in $(X, \tau)$ for every open subset $\mathrm{V}$ of $(\mathrm{Y}, \sigma)$.

xi) Strongly-continuous $[6]$, if $f^{-1}(V)$ is clopen in $(X, \tau)$ for every subset $V$ of $(Y, \sigma)$.

Lemma 2.7 Let $(X, \tau)$ be any topological space, in which

i) Every semi-closed set is srw-closed set in $X[10]$.

ii) Every $\alpha$ rw-closed set is srw-closed set in $\mathrm{X}[10]$.

iii) Every srw-closed set is gs-closed set in $\mathrm{X}[10]$.

iv) Every srw-closed set is gsp-closed set in $\mathrm{X}[10]$. 
Lemma 2.8 If A and B are subsets of a space X. Then [11]

i) $\operatorname{srw}-\operatorname{cl}(\mathrm{X})=\mathrm{X}$ and $\operatorname{srw}-\operatorname{cl}(\varnothing)=\emptyset$.

ii) $\mathrm{A} \subset \operatorname{srw}-\mathrm{cl}(\mathrm{A})$.

iii) If $A \subset B$ then $\operatorname{srw}-\operatorname{cl}(A) \subset \operatorname{srw}-c l(B)$.

Remark 2.9 i) srw-closure of a set A is not always srw-closed set [11]. ii) If $A \subset X$ is srw-closed, then $\operatorname{srw}-\operatorname{cl}(A)=A[11]$.

Lemma 2.10 Let $x \in X$, then $x$ is srw-cl(A) if and only if $V \cap A \neq \emptyset$ for every srw-open set $V$ containing $x$ [11].

Lemma 2.10 A function $f:(X, \tau) \rightarrow(Y, \sigma)$ is called a rw-irresolute function [3], if the inverse image of every rwclosed set in $(\mathrm{X}, \tau)$ for every rw-closed set $\mathrm{V}$ in $(\mathrm{Y}, \sigma)$.

Lemma 2.11 A subset A of a topological space $X$ is srw-open [11], if and only if $F \subseteq \operatorname{sint}(A)$ whenever $F$ is rwclosed and $\mathrm{F} \subseteq \mathrm{A}$.

Semi-regular Weakly Continuous (briefly srw-continuous) Functions:-

In this section, we introduce the concept of srw-continuous functions in topological spaces and study their relations with various generalized continuous functions. We also discuss some properties of srw-continuous functions.

Definition 3.1 A function $\mathrm{f}:(\mathrm{X}, \tau) \rightarrow(\mathrm{Y}, \sigma)$ is said to be semi-regular weakly continuous (briefly srw-continuous) function, if $f^{-1}(V)$ is srw-closed set of $(X, \tau)$ for every closed set $V$ of $(Y, \sigma)$.

Theorem 3.2 If a function $\mathrm{f}:(\mathrm{X}, \tau) \rightarrow(\mathrm{Y}, \sigma)$ is continuous, then it is srw-continuous, but not conversely.

Proof: Let $F$ be closed subset of $Y$. Since $f$ is continuous, $f^{-1}(F)$ is a closed in $X$. By Corollary 3.8 .2 of [10], every closed set is srw-closed set but not conversely. i.e. $\mathrm{f}^{-1}(\mathrm{~F})$ srw-closed. Therefore $\mathrm{f}$ is srw-continuous.

Example 3.3 Let $X=\{a, b, c, d\}$ with topology $\tau=\{\varnothing,\{a\},\{b, c\},\{a, b, c\}, X\}$ and $Y=\{p, q\}$ with topology $\sigma=$ $\{\varnothing,\{p\},\{q\}, Y\}$. Let function $f:(X, \tau) \rightarrow(Y, \sigma)$ be defined by $f(a)=p, f(b)=q, f(c)=q$ and $f(d)=p$. Now $f^{-1}(\varnothing)=\emptyset, f^{-1}(Y)=X, f^{-1}(\{p\})=\{a, b\}$ and $f^{-1}(\{q\})=\{b, c\}$ are srw-closed sets in X. Hence, $f$ is srwcontinuous function. However, since $\{b, c\}$ is not closed set in $\mathrm{X}$ i.e. $\mathrm{f}$ is not continuous on $\mathrm{X}$.

Theorem 3.4 If a function $\mathrm{f}:(\mathrm{X}, \tau) \rightarrow(\mathrm{Y}, \sigma)$ is $\alpha \mathrm{rw}$-continuous, then it is srw-continuous, but not conversely.

Proof: Let $F$ be closed subset of $Y$. Since $f$ is $\alpha r w$-continuous, $f^{-1}(F)$ is a $\alpha \mathrm{rw}$-closed in $\mathrm{X}$. From theorem 3.2 of [10], every $\alpha \mathrm{rw}$-closed set is srw-closed set but not conversely. i.e. $\mathrm{f}^{-1}(\mathrm{~F})$ srw-closed. Therefore $\mathrm{f}$ is srwcontinuous.

Example 3.5 Let $X=\{a, b, c, d\}$ with topology $\tau=\{\varnothing,\{a\},\{b, c\},\{a, b, c\}, X\}$ and $Y=\{1,2\}$ with topology $\sigma=$ $\{\varnothing,\{1\},\{2\}, Y\}$. Let function $\mathrm{f}:(\mathrm{X}, \tau) \rightarrow(\mathrm{Y}, \sigma)$ be defined by $\mathrm{f}(\mathrm{a})=1, \mathrm{f}(\mathrm{b})=\mathrm{f}(\mathrm{c})=2$ and $\mathrm{f}(\mathrm{d})=1$. Nowf ${ }^{-1}(\varnothing)=$ $\emptyset, \mathrm{f}^{-1}(\mathrm{Y})=\mathrm{X}, \mathrm{f}^{-1}(\{1\})=\{\mathrm{a}, \mathrm{d}\}$ and $\mathrm{f}^{-1}(\{2\})=\{\mathrm{b}, \mathrm{c}\}$ are srw-closed sets in $\mathrm{X}$. Hence, $\mathrm{f}$ is srw-continuous function. However, since $\{b, c\}$ is not $\alpha$ rw-closed set in $X$ i.e. $f$ is not $\alpha$ rw-continuous on $X$.

Theorem 3.6 If a function $\mathrm{f}:(\mathrm{X}, \tau) \rightarrow(\mathrm{Y}, \sigma)$ is semi-continuous, then it is srw-continuous, but not conversely.

Proof: Let $F$ be closed subset of $Y$. Since $f$ is semi-continuous, $f^{-1}(F)$ is a semi-closed in $X$. From theorem 3.6 of [10], every semi-closed set is srw-closed set but not conversely. i.e. $f^{-1}(F)$ srw-closed. Therefore $f$ is srwcontinuous.

Example 3.7 Let $X=\{a, b, c, d\}$ with topology $\tau=\{\varnothing,\{a\},\{b, c\},\{a, b, c\}, X\}$ and $Y=\{1,2\}$ with topology $\sigma=$ $\{\varnothing,\{1\}, Y\}$. Let function $\mathrm{f}:(\mathrm{X}, \tau) \rightarrow(\mathrm{Y}, \sigma)$ be defined by $\mathrm{f}(\mathrm{a})=2, \mathrm{f}(\mathrm{b})=1, \mathrm{f}(\mathrm{c})=2$ and $\mathrm{f}(\mathrm{d})=2$. Now $\mathrm{f}^{-1}(\varnothing)=$ $\emptyset, \mathrm{f}^{-1}(\mathrm{Y})=\mathrm{X}$ and $\mathrm{f}^{-1}(\{2\})=\{\mathrm{a}, \mathrm{c}, \mathrm{d}\}$ are srw-closed sets in $\mathrm{X}$. Hence, $\mathrm{f}$ is srw-continuous function. However, since $\{\mathrm{a}, \mathrm{c}, \mathrm{d}\}$ is not semi-closed set in $\mathrm{X}$ i.e. $\mathrm{f}$ is not semi-continuous on $\mathrm{X}$.

Corollary $3.8 \mathrm{i}$ ) If a function $\mathrm{f}:(\mathrm{X}, \tau) \rightarrow(\mathrm{Y}, \sigma)$ is $\alpha$-continuous, then it is srw-continuous.

ii) If a function $\mathrm{f}:(\mathrm{X}, \tau) \rightarrow(\mathrm{Y}, \sigma)$ is $\delta$-continuous, then it is srw-continuous.

iii) If a function $f:(X, \tau) \rightarrow(Y, \sigma)$ is $\pi$-continuous, then it is srw-continuous.

iv) If a function $f:(X, \tau) \rightarrow(Y, \sigma)$ is completely continuous, then it is srw-continuous.

Proof:i) Let $F$ be closed subset of $Y$. Since $f$ is $\alpha$-continuous, $\mathrm{f}^{-1}(\mathrm{~F})$ is a $\alpha$-closed in $\mathrm{X}$. From Corollary3.8.1 of $[10], \mathrm{f}^{-1}(\mathrm{~F})$ srw-closed. Therefore $\mathrm{f}$ is srw-continuous. 
ii) Let $\mathrm{F}$ be closed subset of $\mathrm{Y}$. Since $\mathrm{f}$ is $\delta$-continuous, $\mathrm{f}^{-1}(\mathrm{~F})$ is a $\delta$-closed in $\mathrm{X}$. From theorem $3.8 .3[10], \mathrm{f}^{-1}(\mathrm{~F})$ srw-closed. Therefore $\mathrm{f}$ is srw-continuous.

iii) Let $\mathrm{F}$ be closed subset of $\mathrm{Y}$. Since $\mathrm{f}$ is $\pi$-continuous, $\mathrm{f}^{-1}(\mathrm{~F})$ is a $\pi$-closed in $\mathrm{X}$. From Corollary 3.8 .4 of [10], $\mathrm{f}^{-1}(\mathrm{~F})$ srw-closed. Therefore $\mathrm{f}$ is srw-continuous.

iv) Let $\mathrm{F}$ be closed subset of $\mathrm{Y}$. Since $\mathrm{f}$ is completely continuous, $\mathrm{f}^{-1}(\mathrm{~F})$ is a completely closed in $\mathrm{X}$. From Corollary3.8.5 of [10], $\mathrm{f}^{-1}(\mathrm{~F})$ srw-closed. Therefore $\mathrm{f}$ is srw-continuous.

Remark 3.9 The converse of Corollary need not be true as shown in the following example.

Let $X=\{a, b, c, d\}$ with topology $\tau=\{\varnothing,\{a\},\{b, c\},\{a, b, c\}, X\}$ and $Y=\{p, q\}$ with topology $\sigma=\{\varnothing,\{p\},\{q\}, Y\}$. Let function $f:(X, \tau) \rightarrow(Y, \sigma)$ be defined by $f(a)=p, f(b)=q, f(c)=q$ and $f(d)=p$. Now $f^{-1}(\varnothing)=\emptyset, f^{-1}(Y)=$ $\mathrm{X}, \mathrm{f}^{-1}(\{\mathrm{p}\})=\{\mathrm{a}, \mathrm{b}\}$ and $\mathrm{f}^{-1}(\{\mathrm{q}\})=\{\mathrm{b}, \mathrm{c}\}$ are srw-closed sets in $\mathrm{X}$. Hence, $\mathrm{f}$ is srw-continuous function. However, since

i) $\{b, c\}$ is not $\alpha$-closed set in $X$ i.e. $f$ is not $\alpha$-continuous on $X$.

ii) $\{b, c\}$ is not $\delta$-closed set in $X$ i.e. $f$ is not $\delta$-continuous on $X$.

iii) $\{b, c\}$ is not $\pi$-closed set in $X$ i.e. $f$ is not $\pi$-continuous on $X$.

iv) $\{b, c\}$ is not regular closed set in $X$ i.e. $f$ is not completely continuous on $X$.

Theorem 3.10 If a function $\mathrm{f}:(\mathrm{X}, \tau) \rightarrow(\mathrm{Y}, \sigma)$ is srw-continuous, then it is gs-continuous, but not conversely.

Proof: Let $F$ be any closed subset of $Y$. Since $f$ is srw-continuous, $f^{-1}(F)$ is a srw-closed in X. From theorem 3.4 of [10], every srw-closed set is gs-closed set but not conversely. i.e. $\mathrm{f}^{-1}(\mathrm{~F}) \mathrm{gs}$-closed. Therefore $\mathrm{f}$ is gs-continuous.

Example 3.11 Let $X=\{a, b, c, d\}$ with topology $\tau=\{\varnothing,\{a\},\{b, c\},\{a, b, c\}, X\}$ and $Y=\{1,2,3\}$ with topology $\sigma=$ $\{\varnothing,\{1\}, \mathrm{Y}\}$. Let function $\mathrm{f}:(\mathrm{X}, \tau) \rightarrow(\mathrm{Y}, \sigma)$ be defined by $\mathrm{f}(\mathrm{a})=1, \mathrm{f}(\mathrm{b})=1, \mathrm{f}(\mathrm{c})=2$ andf $(\mathrm{d})=3$. $\operatorname{Nowf}^{-1}(\varnothing)=$ $\emptyset, \mathrm{f}^{-1}(\mathrm{Y})=\mathrm{X}$ and $\mathrm{f}^{-1}(\{2,3\})=\{\mathrm{c}, \mathrm{d}\}$ are gs-closed sets in $\mathrm{X}$. Hence, $\mathrm{f}$ is gs-continuous function. However, since $\{c, d\}$ is not srw-closed set in $X$ i.e. $f$ is not srw-continuous on $X$.

Corollary 3.12 If a function $f:(X, \tau) \rightarrow(Y, \sigma)$ is srw-continuous then it is gs-continuous.

Proof: Let $F$ be any closed subset of $Y$. Since $f$ is srw-continuous, $f^{-1}(F)$ is a srw-closed in $X$. From theorem 3.10 of [10], $\mathrm{f}^{-1}(\mathrm{~F})$ gsp-closed. Therefore $\mathrm{f}$ is gsp-continuous.

Remark 3.13 The converse of Theorem 3.12 need not be true as shown from example 3.11 i.e. $\mathrm{f}^{-1}(\{2,3\})=\{c, d\}$ is gsp-closed set in X. Hence, $f$ is gsp-continuous function. However, since $\{c, d\}$ is not srw-closed set in $X$ i.e. $f$ is not srw-continuous on $\mathrm{X}$.

Examples can be constructed to see that the concepts of g-continuity, w-continuity and $\alpha w$-continuity are independent with the concept of srw-continuity. Thus the above discussion leads to the following implication diagram.

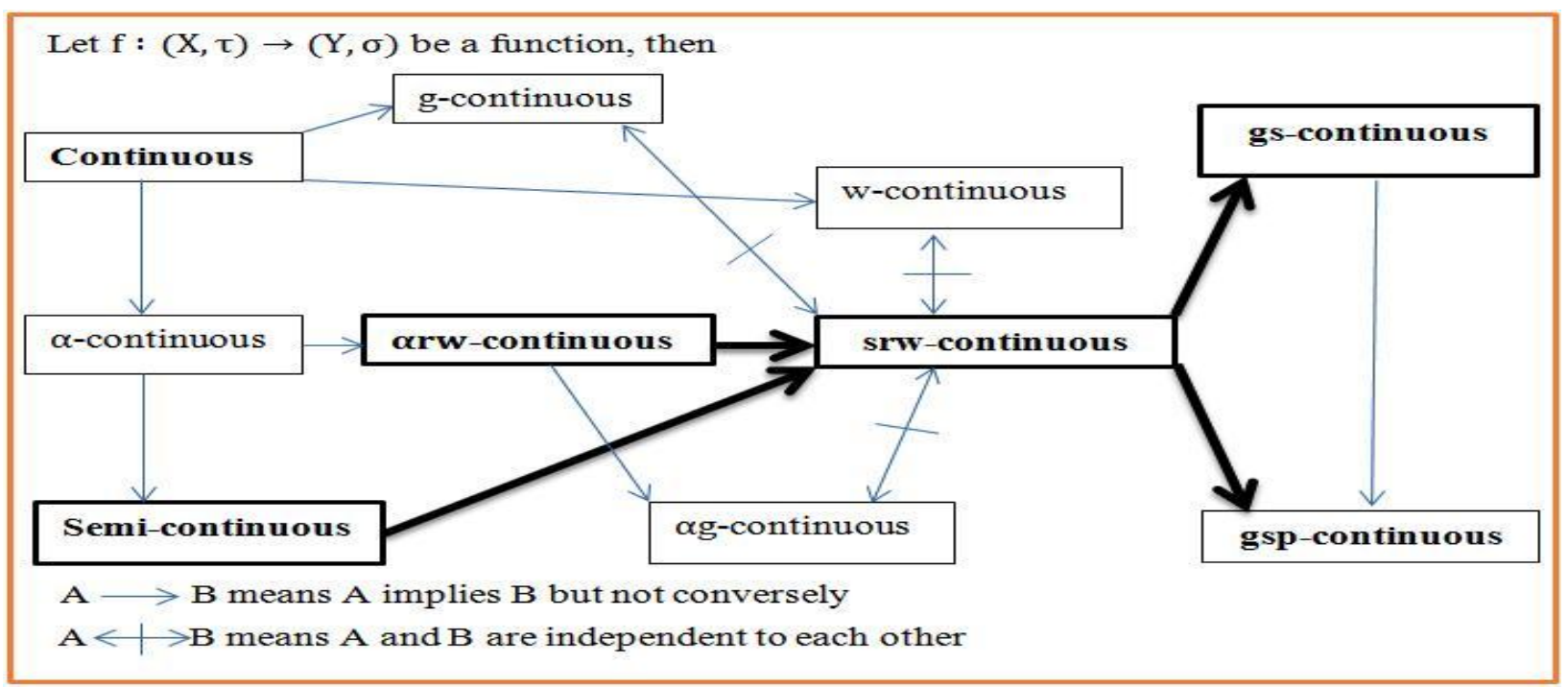


Theorem 3.14 Let $\mathrm{f}:(\mathrm{X}, \tau) \rightarrow(\mathrm{Y}, \sigma)$ be a function, then the following are equivalent.

i) $\mathrm{f}$ is srw-continuous.

ii) The inverse image of each open set in $Y$ is srw-open in $X$.

iii) The inverse image of each closed set in $Y$ is srw-closed in $X$.

Proof: Suppose i) holds. Let $G$ be an open set in $Y$. Then $Y \backslash G$ is closedin $Y$. By (i) $f^{-1}(Y \backslash G)$ is srw-closed set in $X$. But $f^{-1}(Y \backslash G)=X \backslash f^{-1}(G)$ which is srw-closed set in $X$. Therefore $f^{-1}(G)$ is srw-open set in $X$. This proves (i) $\Rightarrow$ (ii).

The implications (ii) $\Rightarrow$ (iii) and (iii) $\Rightarrow$ (i) obviously.

Theorem 3.15 If a function $\mathrm{f}:(\mathrm{X}, \tau) \rightarrow(\mathrm{Y}, \sigma)$ is $\alpha \mathrm{rw}$-irresolute, then it is srw-continuous but not conversely.

Proof: Suppose, a function $\mathrm{f}:(\mathrm{X}, \tau) \rightarrow(\mathrm{Y}, \sigma)$ is $\alpha \mathrm{rw}$-irresolute and let $\mathrm{G}$ is any open set in $\mathrm{Y}$. Then $\mathrm{G}$ is $\alpha \mathrm{rw}$-open set in $\mathrm{Y}$ [12]. Since $\mathrm{f}$ is $\alpha \mathrm{rw}$-irresolute $\mathrm{f}^{-1}(\mathrm{G})$ is $\alpha \mathrm{rw}$-open set in $\mathrm{X}$ and hence srw-open in $\mathrm{X}$ [11]. Thus $\mathrm{f}$ is srwcontinuous.

Example 3.16 Let $X=\{a, b, c, d\}$ with topology $\tau=\{\varnothing,\{a\},\{b, c\},\{a, b, c\}, X\}$ and $Y=\{x, y\}$ with topology $\sigma=$ $\{\varnothing,\{x\},\{y\}, Y\}$. Let function $f:(X, \tau) \rightarrow(Y, \sigma)$ be defined by $f(a)=f(d)=x, f(b)=f(c)=y$. Now $f^{-1}(\varnothing)=\emptyset$, $\mathrm{f}^{-1}(\mathrm{Y})=\mathrm{X}, \mathrm{f}^{-1}(\{\mathrm{x}\})=\{\mathrm{a}, \mathrm{d}\}$ and $\mathrm{f}^{-1}(\{\mathrm{y}\})=\{\mathrm{b}, \mathrm{c}\}$ are gs-closed sets in $\mathrm{X}$. Hence, $\mathrm{f}$ is gs-continuous function. However, since $\{c, d\}$ is not srw-closed set in $X$ i.e. $f$ is not srw-continuous on $X$.

Corollary 3.17 If a function $\mathrm{f}:(\mathrm{X}, \tau) \rightarrow(\mathrm{Y}, \sigma)$ is irresolute, then it is srw-continuous.

Proof: Follows from definition 2.6. (i), 3.6[10] and 3.15.

Theorem 3.18 If a function $\mathrm{f}:(\mathrm{X}, \tau) \rightarrow(\mathrm{Y}, \sigma)$ is srw-continuous and $\mathrm{g}:(\mathrm{Y}, \sigma) \rightarrow(\mathrm{Z}, \gamma)$ is continuous, then their composition $\mathrm{g} \circ \mathrm{f}:(\mathrm{X}, \tau) \rightarrow(\mathrm{Z}, \gamma)$ is srw-continuous.

Proof: Let $\mathrm{F}$ be any closed set in $\mathrm{Z}$, since $\mathrm{g}$ is continuous then $\mathrm{g}^{-1}(\mathrm{~F})$ is closed set in $\mathrm{Y}$. Again $\mathrm{f}$ is srw-continuous then $f^{-1}\left(g^{-1}\right)(F)=(g \circ f)^{-1}(F)$ is a srw-continuous in $X$. Hence $(g \circ f)$ is srw-continuous.

Theorem 3.19 Let $(X, \tau),(Y, \sigma)$ and $(Z, \gamma)$ are topological spaces such that $f:(X, \tau) \rightarrow(Y, \sigma)$ and $g:(Y, \sigma) \rightarrow(Z, \gamma)$ are srw-continuous functions and $\mathrm{Y}$ be a topological space where "every srw-closed subset is closed". Then the function $\mathrm{g} \circ \mathrm{f}:(\mathrm{X}, \tau) \rightarrow(\mathrm{Z}, \gamma)$ is srw-continuous.

Proof: Let $\mathrm{F}$ be closed set in Z. Since $\mathrm{g}$ is srw-continuous function, $\mathrm{g}^{-1}(\mathrm{~F})$ is srw-closed set in Y. By hypothesis, every srw-closed set in $Y$ is closed i.e. $g^{-1}(F)$ is closed set in $Y$. Since $f$ is srw-continuous, $f^{-1}\left(g^{-1}\right)(F)$ is srwclosed set in $X$. But $f^{-1}\left(g^{-1}\right)(F)=(g \circ f)^{-1}(F)$ and hence $(g \circ f)$ is srw-continuous.

\section{Semi-regular Weakly Irresolute (briefly srw-Irresolute) Functions:-}

In this section, we introduce Semi-Regular Weakly irresolute functions and their properties are discussed.

Definition 4.1 A function $\mathrm{f}:(\mathrm{X}, \tau) \rightarrow(\mathrm{Y}, \sigma)$ is called srw-irresolute, if $\mathrm{f}^{-1}(\mathrm{~V})$ is srw-closed set in $\mathrm{X}$ for every srwclosed subset V of Y.

Theorem 4.2 A function $\mathrm{f}:(\mathrm{X}, \tau) \rightarrow(\mathrm{Y}, \sigma)$ is srw-irresolute, if and only if for every srw-open $G$ of $Y, f^{-1}(G)$ is srwopen in X.

Proof: Necessary part- If function $f:(X, \tau) \rightarrow(Y, \sigma)$ is srw-irresolute, then for every srw-closed set $F$ of $Y, f^{-1}(F)$ is srw-closed in $X$. If $G$ is any srw-open subset of $Y$ then $G^{c}$ is srw-closed. Thus $f^{-1}\left(G^{c}\right)$ is srw-closed, but $f^{-1}\left(G^{c}\right)$ is srw-closed set then $f^{-1}\left(G^{c}\right)=\left(f^{-1}(G)\right)^{c}$. So that $f^{-1}(G)$ is srw-open in $X$.

Sufficient part- If for all srw-open subset $G$ of $Y, f^{-1}(G)$ is srw-open in $X$ and if $F$ is any srw-closed subset of $Y$, then $F^{c}$ is srw-open. Also $f^{-1}\left(F^{c}\right)=\left(f^{-1}(G)\right)^{c}$ is srw-open. Thus $f^{-1}(F)$ is srw-closed set in $X$.

Theorem 4.3 A function $\mathrm{f}:(\mathrm{X}, \tau) \rightarrow(\mathrm{Y}, \sigma)$ is srw-irresolute and then it is srw-continuous, but not conversely.

Proof: Let V be any closed set in Y. But V is srw-closed set in Y [10]. As F is srw-irresolute, $\mathrm{f}^{-1}(\mathrm{~V})$ is srw-closed set in $\mathrm{X}$. Therefore, $\mathrm{f}$ is srw-continuous function.

Example 4.4 Let $\mathrm{X}=\mathrm{Y}=\{\mathrm{a}, \mathrm{b}, \mathrm{c}, \quad \mathrm{d}\} \quad$ with topology $\tau=\{\varnothing,\{a\},\{b, c\},\{a, b, c\}, X\}$ and $\sigma=\{\varnothing,\{a\},\{b\},\{a, b\},\{a, b, c\}, Y\}$. Let function $f:(X, \tau) \rightarrow(Y, \sigma)$ be defined by $f(a)=b, f(b)=c, f(c)=d$ and $f(d)=a$. Since, $f$ is srw-continuous but not srw-irresolute functions. However, since $\{c, d\}$ is srw-open set in $Y$, $\mathrm{f}^{-1}(\{\mathrm{a}, \mathrm{d}\})=\{\mathrm{c}, \mathrm{d}\}$ which is not srw-open set in $\mathrm{X}$ i.e. $\mathrm{f}$ is not srw-irresolute function.

Theorem 4.5 If functions $f:(X, \tau) \rightarrow(Y, \sigma)$ and $g:(Y, \sigma) \rightarrow(Z, \gamma)$ are both srw-irresolute, then $g \circ f:(X, \tau) \rightarrow(Z, \gamma)$ is srw-irresolute function.

Proof: If $A \subset Z$ is srw-open set, then $g^{-1}(A)$ is srw-open and $f^{-1}\left(g^{-1}(A)\right)$ is srw-open set. Since $g$ and $f$ are srwirresolute, thus $(g \circ f)^{-1}(A)=f^{-1}\left(g^{-1}(A)\right)$ is srw-open and $g \circ f$ is srw-irresolute. 
Theorem 4.6 Let $(\mathrm{X}, \tau),(\mathrm{Y}, \sigma) \operatorname{and}(\mathrm{Z}, \gamma)$ are any topological spaces. Let $\mathrm{f}:(\mathrm{X}, \tau) \rightarrow(\mathrm{Y}, \sigma)$ and $\mathrm{g}:(\mathrm{Y}, \sigma) \rightarrow(\mathrm{Z}, \gamma)$ are any srw-irresolute and srw-continuous functions respectively, then their composition $g \circ f:(X, \tau) \rightarrow(Z, \gamma)$ is srwcontinuous function.

Proof: Let V be an open set in Z. Consider $(g \circ f)^{-1}(V)=\left(f^{-1} \circ g^{-1}\right)(V)=f^{-1}(U)$, where $U$ is $g^{-1}(V)$ is srw-open set in $\mathrm{Y}$, as $\mathrm{g}$ is srw-continuous. Since $\mathrm{f}$ is srw-irresolute and $\mathrm{f}^{-1}(\mathrm{U})$ is srw-open set in $\mathrm{X}$. Thus $(\mathrm{g} \circ \mathrm{f})$ is srwcontinuous function.

Theorem 4.7 Let function $\mathrm{f}:(\mathrm{X}, \tau) \rightarrow(\mathrm{Y}, \sigma)$ is a rw-irresolute and semi-closed. Then $\mathrm{f}$ maps a srw-closed set in $\mathrm{X}$ into a srw-closed set in Y.

Proof: Let $A$ be srw-closed set in $X$ and $f(A) \subseteq U$, where $U$ is rw-open set in $Y$. Then $A \subseteq f^{-1}(U)$. Since $F$ is rwirresolute, $f^{-1}(U)$ is rw-open in $X$. Since $A$ is srw-closed, $\operatorname{scl}(A) \subseteq f^{-1}(U)$ implies $f(\operatorname{scl}(A)) \subseteq U$. Since $f$ is semiclosed $\mathrm{f}(\operatorname{scl}(\mathrm{A}))$ is semi-closed which implies $\operatorname{scl}(\mathrm{f}(\mathrm{A})) \subseteq \operatorname{scl}(\mathrm{f}(\operatorname{scl}(\mathrm{A})))=\mathrm{f}(\operatorname{scl}(\mathrm{A})) \subseteq \mathrm{U}$. By the definition of srw-closed set, $\mathrm{f}(\mathrm{A})$ is srw-closed set in $\mathrm{Y}$.

Definition 4.8 A space $(X, \tau)$ is said to be $\tau_{\text {srw }}$-space, if every srw-closed set is closed set in $X$

Theorem 4.9 Let function $\mathrm{f}:(\mathrm{X}, \tau) \rightarrow(\mathrm{Y}, \sigma)$ is a srw-continuous and $\tau_{\text {srw }}=\tau$, then $\mathrm{f}$ is srw-irresolute function.

Proof: Let $F$ is srw-closed set in $Y$. Then from definition [ $\tau_{\text {srw }}$-space], $F$ is closed in Y. Since $f$ is srw-continuous, using definition of srw-irresolute $\mathrm{f}^{-1}(\mathrm{~F})$ is srw-closed in $\mathrm{X}$. Therefore $\mathrm{f}$ is srw-irresolute.

Theorem 4.10 Let function $\mathrm{f}:(\mathrm{X}, \tau) \rightarrow(\mathrm{Y}, \sigma)$ is a srw-irresolute and $\tau_{\text {srw }}=\tau$, then $\mathrm{f}$ is srw-continuous function.

Proof: Let F be closed set in Y. Then F is srw-closed set in Y. Since f is srw-irresolute, using definition of srwirresolute functionf ${ }^{-1}(F)$ is srw-closed in $X$. Since $\tau_{\text {srw }}=\tau, f^{-1}(F)$ is closed in $X$. Therefore $f$ is continuous.

Perfectly Semi-regular Weakly Continuous and Strongly Semi-regular Weakly Continuous (briefly perfectly srw-continuous and strongly srw-continuous resp.) Functions:-

Balachandran et. al. introduced the concept of generalized continuous functions of a topological space but strong forms of continuous functions and perfect continuous functions were introduced and discussed by T. Noiri. In this section we introduce and discuss new forms of perfectly srw- continuous and perfectly continuous functions.

Definition 5.1 A function $\mathrm{f}:(\mathrm{X}, \tau) \rightarrow(\mathrm{Y}, \sigma)$ is said to be perfectly srw-continuous if the inverse image of every srwopen set in $(Y, \sigma)$ is both open and closed i.e. clopen in $(X, \tau)$.

Definition 5.2 Let a function $f:(X, \tau) \rightarrow(Y, \sigma)$ is said to be strongly srw-continuous if the inverse image of every srw-open set in $(Y, \sigma)$ is open in $(X, \tau)$.

Theorem 5.3 If a function $\mathrm{f}:(\mathrm{X}, \tau) \rightarrow(\mathrm{Y}, \sigma)$ is strongly srw-continuous function iff the inverse image of every srwclosed set in $(\mathrm{Y}, \sigma)$ is closed in $(\mathrm{X}, \tau)$.

Proof: Suppose that $\mathrm{f}$ is strongly srw-continuous. Let $\mathrm{F}$ be any srw-closed set in $(\mathrm{Y}, \sigma)$. Then $\mathrm{F}^{\mathrm{c}}$ is srw-open set in $(Y, \sigma)$. Since $f$ is strongly srw-continuous, $f^{-1}\left(F^{c}\right)$ is open in $(X, \tau)$. But $f^{-1}\left(F^{c}\right)=X-f^{-1}(F)$, hence $f^{-1}(F)$ is closed set in $(\mathrm{X}, \tau)$.

Conversely, assume that the inverse image of every srw-closed set in $(Y, \sigma)$ is closed in $(X, \tau)$. Let $G$ be any srwopen in $(Y, \sigma)$ and then $G^{c}$ is srw-closed set in $(Y, \sigma)$. By the assumption $f^{-1}\left(G^{c}\right)=X-f^{-1}(G)$, which implies that $f^{-1}(G)$ is open set in $(X, \tau)$. Therefore, $f$ is strongly srw-continuous function.

Theorem 5.4 If a function $\mathrm{f}:(\mathrm{X}, \tau) \rightarrow(\mathrm{Y}, \sigma)$ is perfectly srw-continuous function iff the inverse image of every srwclosed set in $(Y, \sigma)$ is both open and closed in $(X, \tau)$.

Proof: Suppose that $f$ is perfectly srw-continuous. Let $F$ be any srw-closed set in $(Y, \sigma)$. Then $F^{c}$ is srw-open in $(Y, \sigma)$, since $f$ is perfectly srw-continuous, $\mathrm{f}^{-1}\left(\mathrm{~F}^{\mathrm{c}}\right)$ is both open closed sets in $(\mathrm{X}, \tau)$.

Conversely, assume that the inverse image of every srw-closed set in $(\mathrm{Y}, \sigma)$ is both open and closed set in $(\mathrm{X}, \tau)$. Let $\mathrm{G}$ be any srw-open set in $(Y, \sigma)$. Then $\mathrm{G}^{\mathrm{c}}$ is srw-closed in $(\mathrm{Y}, \sigma)$. By the assumption, $\mathrm{f}^{-1}\left(\mathrm{G}^{\mathrm{c}}\right)$ is both open and closed in $(X, \tau)$.

Theorem 5.5 If a function $\mathrm{f}:(\mathrm{X}, \tau) \rightarrow(\mathrm{Y}, \sigma)$ is strongly continuous function, then it is perfectly srw-continuous function, but not conversely. 
Proof: Let A be a srw-open set in $(Y, \sigma)$, then $f^{-1}(A)$ is both open and closed. Since $f$ is strongly continuous, then $f$ is perfectly srw-continuous.

Example 5.6 Let $X=\{a, b, c, d\}, Y=\{p, q, r\}$ with topologies $\tau=\{\varnothing,\{a\},\{b, c\}, X\}$ and $\sigma=\{\varnothing,\{p, q\}, Y\}$. Let a function $\mathrm{f}:(\mathrm{X}, \tau) \rightarrow(\mathrm{Y}, \sigma)$ defined by $\mathrm{f}(\mathrm{a})=\mathrm{r}, \mathrm{f}(\mathrm{b})=\mathrm{p}$ and $\mathrm{f}(\mathrm{c})=\mathrm{q}$. Then $\mathrm{f}$ is perfectly srw-continuous but not strongly-continuous, since for $\{p\}$ is any subset of $(Y, \sigma)$ then $f^{-1}(\{p\})=\{b\}$ which is not clopen in $(X, \tau)$ i.e. $f$ is not strongly-continuous.

Theorem 5.7 If a function $\mathrm{f}:(\mathrm{X}, \tau) \rightarrow(\mathrm{Y}, \sigma)$ is strongly srw-continuous function, then it is continuous function, but not conversely.

Proof: Assume that $\mathrm{f}$ is strongly srw-continuous function. Let $\mathrm{G}$ be any open set in $(\mathrm{Y}, \sigma)$. Since every open set is srw-open set, hence $G$ is srw-open set in $(Y, \sigma)$. Since $f$ is strongly srw-continuous then $f^{-1}(G)$ is open in $(X, \tau)$. Therefore $\mathrm{f}$ is continuous.

Example 5.8 Let $\mathrm{X}=\mathrm{Y}=\quad\{\mathrm{a}, \quad \mathrm{b}, \quad \mathrm{c}, \quad \mathrm{d}\} \quad$ with topology $\tau=\{\varnothing,\{\mathrm{a}\},\{\mathrm{b}, \mathrm{c}\},\{\mathrm{a}, \mathrm{b}, \mathrm{c}\}, \mathrm{X}\}$ and $\sigma=\{\varnothing,\{a\},\{b\},\{a, b\},\{a, b, c\}, Y\}$. Let a function $f:(X, \tau) \rightarrow(Y, \sigma)$ defined by $f(a)=b, f(b)=a, f(c)=d$ and $\mathrm{f}(\mathrm{d})=\mathrm{c}$.Then $\mathrm{f}$ is continuous function but not strongly srw-continuous. However, since $\{\mathrm{c}\}$ is srw-open set in $\mathrm{Y}$ but $\mathrm{f}^{-1}(\{\mathrm{c}\})=\{\mathrm{d}\}$ is not open set in $\mathrm{X}$ i.e. $\mathrm{f}$ is not strongly srw-continuous.

Theorem 5.9 Every strongly srw-continuous function is srw-continuous function, but not conversely.

Proof: Let A be open in Y, every open is srw-open, A is srw-open in $Y$, since $f$ is strongly srw-continuous, $f^{-1}(A)$ is open in $X$ and therefore $f^{-1}(A)$ is srw-open in $X$. Hence $f$ is srw-continuous.

Example 5.10 Let $X=\{a, b, \quad c, d\}, Y=\{p, q, \quad r, s\}$ with topology $\tau=\{\varnothing,\{a\},\{b, c\},\{a, b, c\}, X\}$ and $\sigma=\{\varnothing,\{p\},\{q\},\{p, q\},\{p, q, r\}, Y\}$. Let a function $f:(X, \tau) \rightarrow(Y, \sigma)$ defined by $f(a)=q, f(b)=p, f(c)=s$ and $\mathrm{f}(\mathrm{d})=\mathrm{r}$. Then $\mathrm{f}$ is srw-continuous but not strongly srw-continuous. However, since $\{\mathrm{p}, \mathrm{q}\}$ is srw-open set in $\mathrm{Y}$ but $\mathrm{f}^{-1}(\{\mathrm{p}, \mathrm{q}\})=\{\mathrm{a}, \mathrm{b}\}$ is not open set in $\mathrm{X}$ i.e. $\mathrm{f}$ is not strongly srw-continuous.

Theorem 5.11 If function $\mathrm{f}:(\mathrm{X}, \tau) \rightarrow(\mathrm{Y}, \sigma)$ is strongly continuous function, then it is strongly srw-continuous function, but not conversely.

Proof: Let $\mathrm{f}$ is strongly srw-continuous function i.e. inverse image of every open set in $(Y, \sigma)$ is open in $(X, \tau)$. Since every open set in $(Y, \sigma)$ is srw-open in $(Y, \sigma)$. Therefore, if $G$ be any srw-open set in $(Y, \sigma)$, then $f^{-1}(G)$ is open set in $(X, \tau)$.

Example 5.12 Let $\mathrm{X}=\mathrm{Y}=\quad\{\mathrm{a}, \quad \mathrm{b}, \quad \mathrm{c}, \quad \mathrm{d}\} \quad$ with topology $\tau=\{\varnothing,\{\mathrm{a}\},\{\mathrm{b}, \mathrm{c}\},\{\mathrm{a}, \mathrm{b}, \mathrm{c}\}, \mathrm{X}\}$ and $\sigma=\{\varnothing,\{a\},\{b\},\{a, b\},\{a, b, c\}, Y\}$. Let a function $f:(X, \tau) \rightarrow(Y, \sigma)$ defined by $f(a)=c, f(b)=a, f(c)=a$ and $f(d)=b$. Then $f$ is strongly srw- continuous but not stronglycontinuous, since $\{a\}$ is srw-open set in $Y$ but $\mathrm{f}^{-1}(\{\mathrm{a}\})=\{\mathrm{b}, \mathrm{c}\}$ is open in $\mathrm{X}$ but not closed set in $\mathrm{X}$ i.e. $\mathrm{f}$ is not strongly continuous.

Theorem 5.13 If function $\mathrm{f}:(\mathrm{X}, \tau) \rightarrow(\mathrm{Y}, \sigma)$ is perfectly srw-continuous function, then it is strongly srw-continuous function, but not conversely.

Proof: Assume that $f$ is perfectly srw-continuous function. Let $G$ be any srw-open set in $(Y, \sigma)$. Since $f$ is perfectly srw-continuous then $f^{-1}(G)$ is clopen in $(X, \tau)$. Therefore, $f$ is strongly srw-continuous function.

Example 5.14 Let $X=Y=\quad\{a, \quad b, \quad c, \quad d\}$ with topology $\tau=\{\varnothing,\{a\},\{b, c\},\{a, b, c\}, X\}$ and $\sigma=\{\varnothing,\{a\},\{b\},\{a, b\},\{a, b, c\}, Y\}$. Let a function $f:(X, \tau) \rightarrow(Y, \sigma)$ defined by $f(a)=b, f(b)=c, f(c)=d$ and $\mathrm{f}(\mathrm{d})=\mathrm{a}$. Then $\mathrm{f}$ is strongly srw-continuous but not perfectly srw-continuous, since for any set $\{\mathrm{b}\}$ in $\mathrm{Y}$ but $\mathrm{f}^{-1}(\{\mathrm{~b}\})=\{\mathrm{a}\}$ which is not clopen set in X i.e. $\mathrm{f}$ is not perfectly srw-continuous.

Definition 5.15 A function $\mathrm{f}:(\mathrm{X}, \tau) \rightarrow(\mathrm{Y}, \sigma)$ is called strongly srw-irresolute if the inverse image of every srw-open set $\mathrm{V}$ of $(\mathrm{Y}, \sigma)$ is open in $(\mathrm{X}, \tau)$.

Definition 5.16 A function $\mathrm{f}:(\mathrm{X}, \tau) \rightarrow(\mathrm{Y}, \sigma)$ is called strongly semi-continuous if the inverse image of every semiopen set $\mathrm{V}$ of $(\mathrm{Y}, \sigma)$ is open in $(\mathrm{X}, \tau)$.

Definition 5.17 A function $\mathrm{f:}(\mathrm{X}, \tau) \rightarrow(\mathrm{Y}, \sigma)$ is called strongly gs-continuous if the inverse image of every gs-open set $\mathrm{V}$ of $(\mathrm{Y}, \sigma)$ is open in $(\mathrm{X}, \tau)$.

Definition 5.18 A function $\mathrm{f}:(\mathrm{X}, \tau) \rightarrow(\mathrm{Y}, \sigma)$ is called perfectly gs-continuous if the inverse image of every gs-open set $\mathrm{V}$ of $(\mathrm{Y}, \sigma)$ is clopen in $(\mathrm{X}, \tau)$. 
Theorem 5.19 If a function $\mathrm{f}:(\mathrm{X}, \tau) \rightarrow(\mathrm{Y}, \sigma)$ is perfectly srw-continuous function, then it is perfectly srwcontinuous function.

Proof: As $\mathrm{f}$ is perfectly gs-continuous, $\mathrm{f}^{-1}(\mathrm{U})$ is both open and closed in $\mathrm{X}$ for each gs-open set in $\mathrm{Y}$. Since every srw-open set in $\mathrm{Y}$ is gs-open in $\mathrm{Y}$, then $\mathrm{f}$ is perfectly srw-continuous function.

Theorem 5.20 If a function $\mathrm{f}:(\mathrm{X}, \tau) \rightarrow(\mathrm{Y}, \sigma)$ is perfectly srw-continuous, then $\mathrm{f}$ is perfectly continuous.

Proof: Let $F$ be any open set of $(Y, \sigma)$, since every open set is srw-open set. We get $F$ is srw-open in $(Y, \sigma)$. By hypothesis, we have $f^{-1}(F)$ is clopen in $(X, \tau)$. Hence $f$ is perfectly continuous.

Theorem 5.21 If a function $\mathrm{f}:(\mathrm{X}, \tau) \rightarrow(\mathrm{Y}, \sigma)$ is strongly srw-continuous, then it is strongly semi-continuous, but not conversely.

Proof: Let $f:(X, \tau) \rightarrow(Y, \sigma)$ be strongly srw-continuous function. Since every open set in $X$ is srw-open set in $X[11]$. Therefore if $A$ is semi-open set in $Y$ then $A$ is srw-open set in $Y$. Hence $f^{-1}(A)$ is open set in $X$.

Example 5.22 Let $\mathrm{X}=\mathrm{Y}=\{\mathrm{a}, \quad \mathrm{b}, \quad \mathrm{c}, \quad \mathrm{d}\} \quad$ with topology $\tau=\{\varnothing,\{\mathrm{a}\},\{\mathrm{b}\},\{\mathrm{a}, \mathrm{b}\},\{\mathrm{a}, \mathrm{b}, \mathrm{c}\}, \mathrm{X}\}$ and $\sigma=\{\varnothing,\{a\},\{b, c\},\{a, b, c\}, Y\}$. Let a function $f:(X, \tau) \rightarrow(Y, \sigma)$ defined by $f(a)=c, f(b)=d, f(c)=$ $a$ and $f(d)=b$. Then $f$ is strongly semi-continuous but not strongly srw-continuous, since for srw-open set $\{a\}$ in $Y$ then $\mathrm{f}^{-1}(\{\mathrm{a}\})=\{\mathrm{c}\}$ which is not open set in $\mathrm{X}$ i.e. $\mathrm{f}$ is not strongly srw-continuous.

Theorem 5.23 If a function $\mathrm{f}:(\mathrm{X}, \tau) \rightarrow(\mathrm{Y}, \sigma)$ is strongly gs-continuous, then it is strongly srw-continuous, but not conversely.

Proof: Let $U$ be any srw-open set in $(Y, \sigma)$. By hypothesis, $f^{-1}(U)$ is open and closed in $(X, \tau)$. Hence $f$ is strongly srw-continuous.

Example 5.24 Let $\mathrm{X}=\mathrm{Y}=\{\mathrm{a}, \quad \mathrm{b}, \quad \mathrm{c}, \quad \mathrm{d}\} \quad$ with topology $\tau=\{\varnothing,\{\mathrm{a}\},\{\mathrm{b}\},\{\mathrm{a}, \mathrm{b}\},\{\mathrm{b}, \mathrm{c}\},\{\mathrm{a}, \mathrm{b}, \mathrm{c}\}, \mathrm{X}\}$ and $\sigma=\{\varnothing,\{a\},\{b, c\},\{a, b, c\}, Y\}$. Let a function $f:(X, \tau) \rightarrow(Y, \sigma)$ defined by $f(a)=f(b)=f(c)=a$ and $f(d)=$ c. Then $\mathrm{f}$ is strongly srw-continuous but not strongly gs-continuous, since for gs-open set $\{\mathrm{a}\}$ in $\mathrm{Y}$ but $\mathrm{f}^{-1}(\{\mathrm{a}\})=\{\mathrm{a}, \mathrm{b}\}$ which is not open set in $\mathrm{X}$ i.e. $\mathrm{f}$ is not strongly gs-continuous.

Theorem 5.25 If a function $\mathrm{f}:(\mathrm{X}, \tau) \rightarrow(\mathrm{Y}, \sigma)$ perfectly srw-continuous, then it is strongly srw-continuous, but not conversely.

Proof: Let $F$ be any srw-open set in $(Y, \sigma)$. By hypothesis, $f^{-1}(F)$ is open and closed in $(X, \tau)$, implies that $f^{-1}(F)$ is closed and open in $(X, \tau)$. Hence $f$ is strongly srw-continuous.

Example 5.26 Let $\mathrm{X}=\mathrm{Y}=\{\mathrm{a}, \quad \mathrm{b}, \quad \mathrm{c}, \quad \mathrm{d}\}$ with topology $\tau=\{\varnothing,\{\mathrm{a}\},\{\mathrm{b}, \mathrm{c}\},\{\mathrm{a}, \mathrm{b}, \mathrm{c}\}, \mathrm{X}\}$ and $\sigma=\{\varnothing,\{a\},\{b\},\{a, b\},\{a, b, c\}, Y\}$. Let a function $f:(X, \tau) \rightarrow(Y, \sigma)$ defined by $f(a)=b, f(b)=c, f(c)=$ dand $\mathrm{f}(\mathrm{d})=\mathrm{a}$. Then $\mathrm{f}$ is strongly srw-continuous but not perfectly srw-continuous, since for the srw-open set $\{\mathrm{b}\}$ in $\mathrm{Y}$ but $\mathrm{f}^{-1}(\{\mathrm{~b}\})=\{\mathrm{a}\}$ which is not clopen set in $\mathrm{X}$ i.e. $\mathrm{f}$ is not perfectly srw-continuous.

From the above discussions properties are summarized in the following diagram.

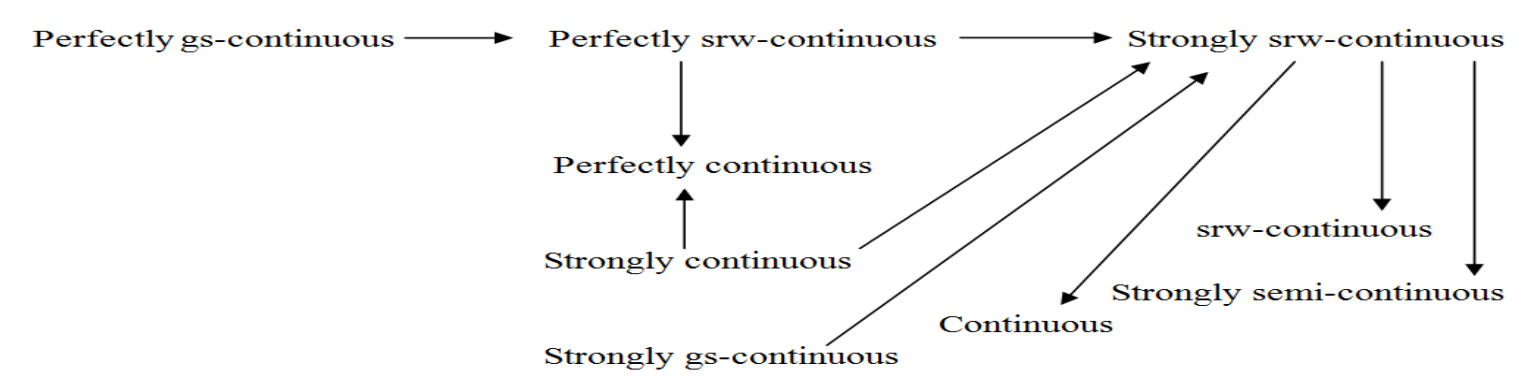

Theorem 5.27 If function $\mathrm{f:}(\mathrm{X}, \tau) \rightarrow(\mathrm{Y}, \sigma)$ is strongly continuous function and $\mathrm{g:}(\mathrm{Y}, \sigma) \rightarrow(\mathrm{Z}, \gamma)$ is srw-continuous function then the composition $\mathrm{g} \circ \mathrm{f:}(\mathrm{X}, \tau) \rightarrow(\mathrm{Z}, \gamma)$ is continuous function.

Proof: Let $G$ be any open set in $(Z, \gamma)$. Since $g$ is srw-continuous, $g^{-1}(G)$ is srw-open set in $(Y, \sigma)$. Since $f$ is strongly srw-continuous, so $\mathrm{f}^{-1}\left(\mathrm{~g}^{-1}(\mathrm{G})\right)=(\mathrm{g} \circ \mathrm{f})^{-1}(\mathrm{G})$ is open $\operatorname{in}(\mathrm{X}, \tau)$. Therefore $\mathrm{g} \circ \mathrm{f}$ is continuous function.

Theorem 5.28 If $\mathrm{f}:(\mathrm{X}, \tau) \rightarrow(\mathrm{Y}, \sigma)$ be a function, let $(\mathrm{X}, \tau)$ be any topological space and $(\mathrm{Y}, \sigma)$ be a $\tau_{\text {srw }}$-space. Then following are equivalent; 
i) $\mathrm{f}$ is strongly srw-continuous.

ii) $\mathrm{f}$ is continuous.

Proof: (i) $\rightarrow$ (ii), Let $U$ be any open set in $(Y, \sigma)$. Since every open set is srw-open, $U$ is srw-open in $(Y, \sigma)$. Then $\mathrm{f}^{-1}(\mathrm{U})$ is open $\operatorname{in}(\mathrm{X}, \tau)$. Hence $\mathrm{f}$ is continuous.

(ii) $\rightarrow$ (i), Let $U$ be any srw-open set in $(Y, \sigma)$. Since $(Y, \sigma)$ be a $\tau_{\text {SRW }}$-space, $U$ is open in $(Y, \sigma)$ and since $f$ is continuous, then $\mathrm{f}^{-1}(\mathrm{U})$ is open $\operatorname{in}(\mathrm{X}, \tau)$. Hence $\mathrm{f}$ is strongly srw-continuous.

Theorem 5.29 Let $\mathrm{f}:(\mathrm{X}, \tau) \rightarrow(\mathrm{Y}, \sigma)$ be a map, both $(\mathrm{X}, \tau)$ and $(\mathrm{Y}, \sigma)$ are $\tau_{\text {srw }}$-space. Then the following are equivalent;

i) $\mathrm{f}$ is srw-irresolute.

ii) $\mathrm{f}$ is strongly srw-continuous.

iii) $\mathrm{f}$ is continuous.

iv) $\mathrm{f}$ is srw-continuous.

Proof: Straight forward.

Theorem 5.30 Let $\mathrm{f}:(\mathrm{X}, \tau) \rightarrow(\mathrm{Y}, \sigma)$ is strongly srw-continuous and $\mathrm{A}$ is open subset of $(\mathrm{X}, \tau)$, then the restriction $\mathrm{f} / \mathrm{A}: \mathrm{A} \rightarrow(\mathrm{Y}, \sigma)$ is strongly srw-continuous.

Proof: Let $V$ be any srw-closed set in $(Y, \sigma)$. Since $f$ is strongly srw-continuous, then $f^{-1}(V)$ is open in $(X, \tau)$. Since $A$ is open $\operatorname{in}(X, \tau)$, then $\left(\frac{f}{A}\right)^{-1}(V)=A \cap f^{-1}(V)$ is open in A. Hence $f / A$ is strongly srw-continuous.

Theorem 5.31 Let $(X, \tau)$ be discrete topological space and $(Y, \sigma)$ be any topological space. Let $f:(X, \tau) \rightarrow(Y, \sigma)$ be a function, then following are equivalent;

i) $\mathrm{f}$ is strongly srw-continuous.

ii) $\mathrm{f}$ is perfectly srw-continuous.

Proof: (i) $\rightarrow$ (ii), Let $U$ be any srw-open set in $(Y, \sigma)$. By hypothesis, $f^{-1}(U)$ is open in $(X, \tau)$. Since $(X, \tau)$ is discrete space, then $f^{-1}(U)$ is also closed $\operatorname{in}(X, \tau)$. Therefore $f^{-1}(U)$ is both open and closed in $(X, \tau)$. Hence $f$ is perfectly srw-continuous.

(ii) $\rightarrow$ (i), Let $U$ be any srw-open set in $(Y, \sigma)$, then $f^{-1}(U)$ is both open and closed in $(X, \tau)$. Hence $f$ is strongly srw-continuous.

Theorem 5.32 If functions $\mathrm{f}:(\mathrm{X}, \tau) \rightarrow(\mathrm{Y}, \sigma)$ and $\mathrm{g}:(\mathrm{Y}, \sigma) \rightarrow(\mathrm{Z}, \gamma)$ are perfectly srw-continuous, then the composition $\mathrm{g} \circ \mathrm{f}:(\mathrm{X}, \tau) \rightarrow(\mathrm{Z}, \gamma)$ is also perfectly srw-continuous function.

Proof: Let $U$ is a srw-open set in $(Z, \gamma)$. Since $g$ is a perfectly srw-continuous, we get $g^{-1}(U)$ is open and closed in $(Y, \sigma)$. As any open set is srw-open in $(X, \tau)$ and $f$ is also strongly srw-continuous, $f^{-1}\left(g^{-1}(U)\right)=(g \circ f)^{-1}(U)$ is both open and closed $\operatorname{in}(\mathrm{X}, \tau)$. Hence $\mathrm{g} \circ \mathrm{f}$ is perfectly srw-continuous.

Theorem 5.33 If $\mathrm{f}:(\mathrm{X}, \tau) \rightarrow(\mathrm{Y}, \sigma)$ and $\mathrm{g:}(\mathrm{Y}, \sigma) \rightarrow(\mathrm{Z}, \gamma)$ are functions, then the composition $\mathrm{g} \circ \mathrm{f}:(\mathrm{X}, \tau) \rightarrow(\mathrm{Z}, \gamma)$ is i) srw-irresolute, if $\mathrm{g}$ is strongly srw-continuous and $\mathrm{f}$ is srw-continuous.

ii) Strongly srw-continuous, if $g$ is perfectly srw-continuous and $\mathrm{f}$ is continuous.

iii) Perfectly continuous, if $\mathrm{g}$ is strongly srw-continuous and $\mathrm{f}$ is perfectly srw-continuous.

Proof: i) Let $U$ be a srw-open set in $(Z, \gamma)$, then $g^{-1}(U)$ is open set in $(Y, \sigma)$. Since $f$ is continuous, $f^{-1}\left(g^{-1}(U)\right)=$ $(\mathrm{g} \circ \mathrm{f})^{-1}(\mathrm{U})$ is open in $(X, \tau)$. Hence $\mathrm{g} \circ \mathrm{f}$ is srw-irresolute.

ii) Let $U$ be a srw-open set in $(Z, \gamma)$, then $g^{-1}(U)$ is both open and closed in $(Y, \sigma)$. Therefore, $f^{-1}\left(g^{-1}(U)\right)=$ $(\mathrm{g} \circ \mathrm{f})^{-1}(\mathrm{U})$ is both open and closed in $(\mathrm{X}, \tau)$. Hence $\mathrm{g} \circ \mathrm{f}$ is strongly srw-continuous.

iii) Let $U$ be a srw-open set in $(Z, \gamma)$, then $g^{-1}(U)$ is both open and closed in $(Y, \sigma)$. By hypothesis, $f^{-1}\left(g^{-1}(U)\right)=$ $(\mathrm{g} \circ \mathrm{f})^{-1}(\mathrm{U})$ is open in $(\mathrm{X}, \tau)$. Hence $\mathrm{g}^{\circ} \mathrm{f}$ is perfectly srw-continuous.

Theorem 5.34 If $\mathrm{f}:(\mathrm{X}, \tau) \rightarrow(\mathrm{Y}, \sigma)$ and $\mathrm{g}:(\mathrm{Y}, \sigma) \rightarrow(\mathrm{Z}, \gamma)$ are strongly srw-continuous functions, then their composition $\mathrm{g} \circ \mathrm{f}:(\mathrm{X}, \tau) \rightarrow(\mathrm{Z}, \gamma)$ is also strongly srw-continuous.

Proof: Let $U$ be a srw-open set in $(Z, \gamma)$. Since $g$ is strongly srw-continuous, we have $g^{-1}(U)$ is open in(Y, $\left.\sigma\right)$. It is srw-open $\operatorname{in}(Y, \sigma)$. As $f$ is also stronglysrw-continuous, $f^{-1}\left(g^{-1}(U)\right)=(g \circ f)^{-1}(U)$ is open in $(X, \tau)$. Hence $g \circ f$ is strongly srw-continuous. 


\section{References:-}

1. I. Arockiarani, Studies on Generalizations of Generalized Closed sets and Maps in Topological Spaces, Ph.D. thesis, Bharathiar Univ. Coimbatore(1997).

2. K. Balachandran, P. Sundaram and H. Maki, Generalized locally closed sets and GLC-continuous functions, Indian J. Pure Appl. Math., 27(1996), 235-244.

3. Dr. S. S. Benchalli and R. S. Wali, Some Topics in General and Fuzzy Topological Spaces, Ph.D. thesis, Karnataka Univ. Dharwad(2006);7-8.

4. R. Devi, K. Balachandran and H. Maki, On Generalized $\alpha$-continuous Maps, Far., East J. Math. Sci. Special Volume Part-I(1997),1-15.

5. J. Dontchev and H. Maki, On sg-closed sets and semi -closed sets, Questions and Answers Gen. Topology; 15(1997),253-266.

6. Kavitamani K., Studies on a New Generalization of Homeomorphisms in Topological Spaces, Ph.D. thesis, Karpagam University Coimbatore(2013)-11-12.

7. N. Levine, Semi Continuous in Topological Spaces, Amer. Math. Monthly, 67(1960), 269.

8. A. S. Mashhour, I. A. Hasanein and S. N. EI-Deeb, $\alpha$-continuous and $\alpha$-open Mapping, Acta.Math. Hung, 41(1983),213-218.

9. N. Palaniappan and K. C. Rao, Regular Generalized Closed Sets, Kyungpook, Math. J. 33(1993), 211-219.

10. R. S. Wali and Basayya B. Mathad, Semi Regular Weakly Closed Sets in Topological Spaces, Int. J. of Mathematical Archive-7(6)(2016),91-97.

11. R. S. Wali and Basayya B. Mathad, Semi Regular Weakly Open Sets in Topological Spaces, Int. J. of Mathematics Trends and Technology-Volume 37, 1Sept.2016.

12. R. S. Wali and Prabhavati S. Mendalgeri, On arw-Continuous and arw-Irresolute Maps in Topological Spaces, IOSR Journal of Mathematics, Vol.10 (6)(2014),14-24. 